\title{
PROPAGATION OF DISTURBANCE IN BOUNDARY LAYER ON PLANAR DELTA WING IN THE STRONG INTERACTION REGIME
}

G. N. Dudin

\author{
Moscow Institute of Physics and Technology (State University)
}

Moscow, Russia

The flow past a planar delta wing is studied for strong interaction between the boundary layer and the outer supersonic flow. The disturbance propagation in the laminar boundary layer is investigated. The characteristic surface associated with induced pressure is found. The results of the theoretical and numerical analysis of disturbance propagation under changes of the relevant parameters of the problem for three-dimensional (3D) flows are presented.

\section{FORMULATION OF THE PROBLEM}

There will be considered the distinctive features of disturbance propagation and the formulation of the boundary value problem for the boundary layer on a plane delta wing in the case in which the interaction with the outer hypersonic flow is strong under the following conditions:

$$
\mathrm{M}_{\infty} \rightarrow \infty, \operatorname{Re}_{0} \rightarrow \infty, \mathrm{M}_{\infty} \delta \rightarrow \infty
$$

Here, $\mathrm{M}_{\infty}$ is the Mach number of the undisturbed inviscid flow; $\operatorname{Re}_{0}=\rho_{\infty} u_{\infty} l / \mu_{0}$ is the Reynolds number; and $\delta=\left(s / \operatorname{Re}_{0}\right)^{1 / 4}$ is the dimensionless boundary layer thickness. The parameter $s=\operatorname{tg} \omega_{0}$ characterizing the transverse-to-longitudinal wing size ratio is of the order $O(1)$. The angle $\omega_{0}$ is the angle which the leading edge makes with the oncoming flow. The results obtained make it possible to draw some interesting conclusions concerning the general 3D flow. A Cartesian coordinate system with origin at the wing nose, the $x^{*} l$-axis aligned with the axis of symmetry of the wing, the $z^{*} s l$-axis normal to the former and lying in the wing plane, and the $y^{*} \delta l$-axis normal to the $x^{*} z^{*}$ plane are introduced (Fig. 1 ).

This is an Open Access article distributed under the terms of the Creative Commons Attribution-Noncommercial License 3.0, which permits unrestricted use, distribution, and reproduction in any noncommercial medium, provided the original work is properly cited. 


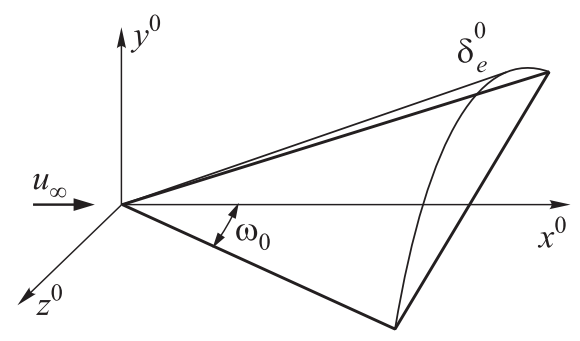

Figure 1 Cartesian coordinate system

The velocity components $u u_{\infty}, v^{*} u_{\infty} \delta / s_{0}$, and $w u_{\infty}$ are directed along the $x^{*}, y^{*}$, and $z^{*}$ axes, respectively. The time, density, pressure, total enthalpy, boundary layer thickness, and viscosity are $t l / u_{\infty}, \rho^{*} \delta^{2} \rho_{\infty}, p^{*} \delta^{2} \rho_{\infty} u_{\infty}^{2}, g u_{\infty}^{2} / 2$, $\delta l \delta_{e}^{*}$, and $\mu \mu_{0}$, where the subscript $\infty$ refers to the dimensional parameters of the oncoming flow and $\mu_{0}$ is the dynamic viscosity determined at the stagnation temperature. It is assumed that the gas is thermodynamically perfect and characterized by a constant specific heat ratio $\gamma$. The Reynolds number is high but not higher than the critical value corresponding to laminar-turbulent transition.

In accordance with the theory of strong interaction, the disturbed flow region can be divided into two subdomains, namely, the shock layer I and the boundary layer II. In subdomain I, the approximate equation is used:

$$
p^{*}=\frac{\gamma+1}{2}\left(\frac{\partial \delta_{e}^{*}}{\partial x^{*}}+\frac{\partial \delta_{e}^{*}}{\partial t}\right)^{2}
$$

which represents the generalization of the tangent wedge formula to the timedependent case [1]. In subdomain II, there is a system of equations for a timedependent 3D boundary layer [2]. For taking account for the singular behavior of the unknown functions in the vicinity of the delta wing vertex and the leading edges, the similarity variables [2] and the Dorodnitsyn variables are introduced:

$$
\left.\begin{array}{c}
\lambda=\int_{0}^{y^{*}} \rho^{*} d y^{*} ; \quad v_{\delta}^{*}=\rho^{*} v^{*}+s \frac{\partial \lambda}{\partial t}+w \frac{\partial \lambda}{\partial z^{*}}+s u \frac{\partial \lambda}{\partial x^{*}} ; \\
u=\frac{\partial F}{\partial \lambda} ; \quad w=\frac{\partial \Phi}{\partial \lambda} ; \\
x^{*}=x ; z^{*}=x z ; \lambda=x^{1 / 4} \sqrt{\frac{2 \gamma}{\gamma-1}\left(1-z^{2}\right)^{1 / 2}} \eta ; \\
F=\sqrt{\frac{2 \gamma}{\gamma-1}} x^{1 / 4} \Theta ; \quad \Phi=\sqrt{\frac{2 \gamma}{\gamma-1}} x^{1 / 4} N ; \\
p^{*}=x^{-1 / 2}\left(1-z^{2}\right)^{-1 / 2} p ; \rho^{*}=x^{-1 / 2} \rho ; \quad \delta_{e}^{*}=x^{3 / 4}\left(1-z^{2}\right)^{3 / 4} \delta_{e} .
\end{array}\right\}
$$


Rewriting the system of dimensionless boundary layer equations and boundary conditions in terms (1) yields:

$$
\left.\begin{array}{c}
A_{0} \frac{\partial u}{\partial t}+A_{1} \frac{\partial u}{\partial x}+A_{2} \frac{\partial u}{\partial z}+v \frac{\partial u}{\partial \eta}=-\frac{\gamma-1}{2 \gamma p} s G\left[\left(1-z^{2}\right)\left(\frac{x}{p} \frac{\partial p}{\partial x}-\frac{1}{2}\right)\right. \\
\left.-z\left(z+\frac{1-z^{2}}{p} \frac{\partial p}{\partial x}\right)\right]+\frac{\partial^{2} u}{\partial \eta^{2}} ; \\
A_{0} \frac{\partial w}{\partial t}+A_{1} \frac{\partial w}{\partial x}+A_{2} \frac{\partial w}{\partial z}+v \frac{\partial w}{\partial \eta} \\
=-\frac{\gamma-1}{2 \gamma p} G\left(z+\frac{1-z^{2}}{p} \frac{\partial p}{\partial z}\right)+\frac{\partial^{2} w}{\partial \eta^{2}} ; \\
A_{0} \frac{\partial g}{\partial t}+A_{1} \frac{\partial g}{\partial x}+A_{2} \frac{\partial g}{\partial z}+v \frac{\partial g}{\partial \eta}=\frac{\gamma-1}{\gamma p^{2}} s x\left(1-z^{2}\right) G \frac{\partial p}{\partial t} \\
+\frac{\partial}{\partial \eta}\left[\frac{1}{\sigma} \frac{\partial g}{\partial \eta}-\frac{1-\sigma}{\sigma} \frac{\partial\left(u^{2}+w^{2}\right)}{\partial \eta}\right] ; \\
v=-\frac{\left(1-z^{2}\right)^{3 / 4}}{p}\left[s\left(\frac{1}{4} \Theta+x \frac{\partial \Theta}{\partial x}-z \frac{\partial \Theta}{\partial z}\right)+\frac{\partial N}{\partial z}\right] ; \\
\delta_{e}=\frac{\sqrt{\gamma-1}}{\sqrt{2 \gamma} p} \int_{0}^{\infty} G d \eta ; \\
p=\frac{\partial+1}{2}\left[\frac{3}{4}\left(1+z^{2}\right) \delta_{e}+x\left(1-\frac{\partial \delta_{e}}{\partial t}\right)-z\left(1-z^{2}\right) \frac{\partial \delta_{e}}{\partial z}\right]^{2}
\end{array}\right\}
$$

where

$$
\begin{aligned}
& u=\left(1-z^{2}\right)^{-1 / 4} \frac{\partial \Theta}{\partial \eta} ; w=\left(1-z^{2}\right)^{-1 / 4} \frac{\partial N}{\partial \eta} ; G=g-u^{2}-w^{2} \\
& A_{0}=\operatorname{sxp}^{-1}\left(1-z^{2}\right) ; A_{1}=A_{0} u ; A_{2}=(w-s u z) p^{-1}\left(1-z^{2}\right) .
\end{aligned}
$$

One may note that in the boundary layer approximation, the equation for the normal momentum component is degenerated; hence follows that the functions $p$ are independent of the coordinate $\eta$. It is assumed that the viscosity-temperature dependence is linear and $\sigma$ is the Prandtl number. The solution of this system must satisfy the boundary conditions at the surface and the outer edge of the boundary layer:

$$
\begin{array}{ll}
\eta=0: & u=w=F=\Phi=0, g=g_{w} \\
\eta \rightarrow \infty: & u \rightarrow 1, w \rightarrow 0, g \rightarrow 1
\end{array}
$$




\section{DETERMINING CHARACTERISTICS SURFACES IN TIME-DEPENDENT THREE-DIMENSIONAL FLOWS}

According [3, 4], the characteristic surfaces are cylindrical surfaces normal to the body surface. A characteristic surface $f(x, z, t)=0$ associated with a function $p(x, z, t)$ is a surface on which some initial data are proposed and the derivative $\partial p / \partial f$ is not determined. The procedure used below is applicable only under conditions in which the pressure distribution in the boundary layer equations is not known beforehand and is determined in the process of solution. For this purpose, the system must involve the additional interaction condition and the additional boundary conditions. After introduction of new variables $x, z, \eta, t$ $\rightarrow f(x, z, t), z, \eta, t$, the boundary value problem takes the form:

$$
\begin{aligned}
& A \frac{\partial u}{\partial f}+A_{0} \frac{\partial u}{\partial t}+A_{2} \frac{\partial u}{\partial z}+v \frac{\partial u}{\partial \eta} \\
& =-\frac{\gamma-1}{2 \gamma p^{2}}\left(1-z^{2}\right) s G\left(x \frac{\partial f}{\partial x}-z \frac{\partial f}{\partial z}\right) \frac{\partial p}{\partial f} \\
& +\frac{\gamma-1}{2 \gamma p} s G\left(\frac{1}{2}\left(1+z^{2}\right)+z \frac{1-z^{2}}{p} \frac{\partial p}{\partial z}\right)+\frac{\partial^{2} u}{\partial \eta^{2}} \text {; } \\
& A \frac{\partial w}{\partial f}+A_{0} \frac{\partial w}{\partial t}+A_{2} \frac{\partial w}{\partial z}+v \frac{\partial w}{\partial \eta} \\
& =-\frac{\gamma-1}{2 \gamma p^{2}}\left(1-z^{2}\right) G \frac{\partial f}{\partial z} \frac{\partial p}{\partial f}-\frac{\gamma-1}{2 \gamma p} G\left(z+\frac{1-z^{2}}{p} \frac{\partial p}{\partial z}\right)+\frac{\partial^{2} w}{\partial \eta^{2}} ; \\
& A \frac{\partial g}{\partial f}+A_{0} \frac{\partial g}{\partial t}+A_{2} \frac{\partial g}{\partial z}+v \frac{\partial g}{\partial \eta} \\
& =\frac{\gamma-1}{\gamma p^{2}}\left(1-z^{2}\right) x s G \frac{\partial f}{\partial t} \frac{\partial p}{\partial f}+\frac{\gamma-1}{\gamma p^{2}}\left(1-z^{2}\right) x s G \frac{\partial p}{\partial t} \\
& +\frac{\partial}{\partial \eta}\left[\frac{1}{\sigma} \frac{\partial g}{\partial \eta}-\frac{1-\sigma}{\sigma} \frac{\partial\left(u^{2}+w^{2}\right)}{\partial \eta}\right] ; \\
& v=-\frac{\left(1-z^{2}\right)^{3 / 4}}{p}\left[\frac{1}{4} s \Theta+s\left(x \frac{\partial f}{\partial x}-z \frac{\partial f}{\partial z}\right) \frac{\partial \Theta}{\partial f}\right. \\
& \left.-s z \frac{\partial \Theta}{\partial x}+\frac{\partial N}{\partial z}+\frac{\partial f}{\partial z} \frac{\partial N}{\partial f}\right] \\
& p=\frac{\gamma+1}{2}\left[\frac{3}{4}\left(1+z^{2}\right) \delta_{e}+\left(1-z^{2}\right)\left(x \frac{\partial \delta_{e}}{\partial t}-z \frac{\partial \delta_{e}}{\partial z}\right)\right. \\
& \left.+\left(1-z^{2}\right)\left[x\left(\frac{\partial f}{\partial x}+\frac{\partial f}{\partial t}\right)-z \frac{\partial f}{\partial z}\right] \frac{\partial \delta_{e}}{\partial f}\right]^{2} ; \\
& \delta_{e}=\frac{\sqrt{\gamma-1}}{\sqrt{2 \gamma} p} \int_{0}^{\infty} G d \eta ; A=A_{0} \frac{\partial f}{\partial t}+A_{1} \frac{\partial f}{\partial x}+A_{2} \frac{\partial f}{\partial z} \text {. }
\end{aligned}
$$


Using the definition of the boundary layer displacement thickness, the derivative on the right-hand side of equation can be expressed as follows:

$$
\frac{\partial \delta_{e}}{\partial f}=\sqrt{\frac{\gamma-1}{2 \gamma}} \frac{1}{p}\left(\int_{0}^{\infty} \frac{\partial G}{\partial f} d \eta-\frac{1}{p} \frac{\partial p}{\partial f} \int_{0}^{\infty} G d \eta\right) .
$$

For determining the derivative $\partial \delta_{e} / \partial f$, the system of equations is transformed into a single equation for the function

$$
R=s\left(x \frac{\partial f}{\partial x}-z \frac{\partial f}{\partial z}\right) \frac{\partial \Theta}{\partial f}+\frac{\partial f}{\partial z} \frac{\partial N}{\partial f} .
$$

Summing the first equation from system (3) multiplied by $s(x \partial f / \partial x-z \partial f / \partial z)$ and the second equation from system (3) multiplied by $\partial f / \partial z$, one obtains the following equation:

$$
Q \frac{\partial R}{\partial \eta}-R \frac{\partial Q}{\partial \eta}+E G \frac{\partial p}{\partial f}=C
$$

where

$$
\begin{aligned}
Q= & s x\left(\frac{\partial f}{\partial t}+u \frac{\partial f}{\partial x}\right)+(w-s u z) \frac{\partial f}{\partial z} \\
E= & \frac{\gamma-1}{2 \gamma p}\left(1-z^{2}\right)^{1 / 4}\left[s^{2}\left(x \frac{\partial f}{\partial x}-z \frac{\partial f}{\partial z}\right)^{2}+\left(\frac{\partial f}{\partial z}\right)^{2}\right] ; \\
C= & \left(1-z^{2}\right)^{-3 / 4} p\left[s\left(x \frac{\partial f}{\partial x}-z \frac{\partial f}{\partial z}\right)\left(L_{2}-L_{3}-L_{4} \frac{\partial u}{\partial \eta}\right)\right. \\
& \left.\quad+\frac{\partial f}{\partial z}\left(M_{2}-M_{3}-L_{4} \frac{\partial w}{\partial \eta}\right)\right] ; \\
L_{2}= & s \frac{\gamma-1}{4 \gamma p} G\left(1+z^{2}+2 z \frac{1-z^{2}}{p} \frac{\partial p}{\partial z}\right)+\frac{\partial^{2} u}{\partial \eta^{2}} ; \\
L_{3}= & \frac{1-z^{2}}{p}\left[s x \frac{\partial u}{\partial t}+(w-s u z) \frac{\partial u}{\partial z}\right] ; \\
L_{4}= & -\frac{\left(1-z^{2}\right)^{3 / 4}}{p}\left(\frac{s}{4} \Theta-s z \frac{\partial \Theta}{\partial z}+\frac{\partial N}{\partial z}\right) ; \\
M_{2}= & -\frac{\gamma-1}{2 \gamma p} G\left(z+\frac{1-z^{2}}{p} \frac{\partial p}{\partial z}\right)+\frac{\partial^{2} w}{\partial \eta^{2}} ; \\
M_{3}= & \frac{1-z^{2}}{p}\left[s x \frac{\partial w}{\partial t}+(w-s u z) \frac{\partial w}{\partial z}\right] .
\end{aligned}
$$


Equation (4) has the following solution:

$$
R=-E Q \frac{\partial p}{\partial f} \int_{0}^{\eta} \frac{G}{Q^{2}} d \eta+Q \int_{0}^{\eta} \frac{C}{Q^{2}} d \eta
$$

which yields the following expressions for the derivatives $\partial u / \partial f, \partial w / \partial f$, and $\partial g / \partial f$ from system (3):

$$
\begin{aligned}
& \frac{\partial u}{\partial f}=\frac{\left(1-z^{2}\right)^{-1 / 4}}{Q}\left[\left(\frac{p}{\left(1-z^{2}\right)^{3 / 4}} L_{1}-E Q \frac{\partial u}{\partial \eta} \int_{0}^{\eta} \frac{G}{Q^{2}} d \eta\right) \frac{\partial p}{\partial f}\right. \\
& \left.+\frac{\partial u}{\partial \eta} Q \int_{0}^{\eta} \frac{C}{Q^{2}} d \eta+L_{5}\right] \\
& \frac{\partial w}{\partial f}=\frac{\left(1-z^{2}\right)^{-1 / 4}}{Q}\left[\left(\frac{p}{\left(1-z^{2}\right)^{3 / 4}} M_{1}-E Q \frac{\partial w}{\partial \eta} \int_{0}^{\eta} \frac{G}{Q^{2}} d \eta\right) \frac{\partial p}{\partial f}\right. \\
& \left.+\frac{\partial w}{\partial \eta} Q \int_{0}^{\eta} \frac{C}{Q^{2}} d \eta+M_{5}\right]^{0} \\
& \frac{\partial g}{\partial f}=\frac{\left(1-z^{2}\right)^{-1 / 4}}{Q}\left[\left(\frac{p}{\left(1-z^{2}\right)^{3 / 4}} K_{1}-E Q \frac{\partial g}{\partial \eta} \int_{0}^{\eta} \frac{G}{Q^{2}} d \eta\right) \frac{\partial p}{\partial f}\right. \\
& \left.+\frac{\partial g}{\partial \eta} Q \int_{0}^{\eta} \frac{C}{Q^{2}} d \eta+K_{5}\right]
\end{aligned}
$$

where

$$
\begin{gathered}
L_{1}=-\frac{\gamma-1}{2 \gamma p^{2}} s\left(1-z^{2}\right) G\left(x \frac{\partial f}{\partial x}-z \frac{\partial f}{\partial z}\right) ; M_{1}=-\frac{\gamma-1}{2 \gamma p^{2}}\left(1-z^{2}\right) G \frac{\partial f}{\partial z} \\
K_{1}=\frac{\gamma-1}{\gamma p^{2}} s x\left(1-z^{2}\right) G \frac{\partial f}{\partial t} \\
K_{2}=\frac{\gamma-1}{\gamma p^{2}}\left(1-z^{2}\right) x s G \frac{\partial p}{\partial t}+\frac{\partial}{\partial \eta}\left[\frac{1}{\sigma} \frac{\partial g}{\partial \eta}-\frac{1-\sigma}{\sigma} \frac{\partial\left(u^{2}+w^{2}\right)}{\partial \eta}\right] ; \\
K_{3}=s x \frac{1-z^{2}}{p} \frac{\partial g}{\partial t}+(w-s u z) \frac{1-z^{2}}{p} \frac{\partial g}{\partial z} \\
K_{5}=\left(K_{2}-K_{3}-L_{4} \frac{\partial g}{\partial \eta}\right) \frac{p}{\left(1-z^{2}\right)^{3 / 4}}
\end{gathered}
$$




$$
\begin{aligned}
L_{5} & =\left(L_{2}-L_{3}-L_{4} \frac{\partial u}{\partial \eta}\right) \frac{p}{\left(1-z^{2}\right)^{3 / 4}} \\
M_{5} & =\left(M_{2}-M_{3}-L_{4} \frac{\partial w}{\partial \eta}\right) \frac{p}{\left(1-z^{2}\right)^{3 / 4}} .
\end{aligned}
$$

Using expressions (5), the following integral is calculated:

$$
\int_{0}^{\infty} \frac{\partial G}{\partial f} d \eta=\int_{0}^{\infty}\left(\frac{\partial g}{\partial f}-2 u \frac{\partial u}{\partial f}-2 w \frac{\partial w}{\partial f}\right) d \eta
$$

taking into to acount that

$$
\int_{0}^{\infty} \frac{\partial G}{\partial \eta}\left(\int_{0}^{\eta} \frac{G}{Q^{2}} d \eta\right) d \eta=-\int_{0}^{\infty} \frac{G^{2}}{Q^{2}} d \eta
$$

Finally, the expression for the derivative of the induced pressure in the direction perpendicular to the characteristic surface is obtained:

$$
\left.\begin{array}{c}
\frac{\partial p}{\partial f}=\frac{p}{I}\left[\sqrt{\frac{2 p}{\gamma+1}-\frac{3}{4}\left(1+z^{2}\right) \delta_{e}-\left(1-z^{2}\right)\left(x \frac{\partial \delta_{e}}{\partial t}-z \frac{\partial \delta_{e}}{\partial z}\right)}\right. \\
-I_{2} \sqrt{\left.\frac{\gamma-1}{2 \gamma} \frac{1-z^{2}}{p}\left(x\left(\frac{\partial f}{\partial x}+\frac{\partial f}{\partial t}\right)-z \frac{\partial f}{\partial z}\right)\right] ;} \\
I=N\left(1-z^{2}\right) \sqrt{\frac{\gamma-1}{2 \gamma}}\left[x\left(\frac{\partial f}{\partial x}+\frac{\partial f}{\partial t}\right)-z \frac{\partial f}{\partial z}\right] ; \\
\times \int_{0}^{\infty} \frac{\gamma-1}{\left[s_{0} x \partial f / \partial t+s_{0} u(x \partial f / \partial x-z \partial f / \partial z)+w \partial f / \partial z\right]^{2}}-\int_{0}^{\infty} G d \eta .
\end{array}\right\}
$$

Returning to variables $\left(x^{*}, \lambda, z^{*}, t\right)$ and introduction of the characteristic surface displacement velocity

$$
\begin{gathered}
a=-\frac{1}{\sqrt{B}} \frac{\partial f}{\partial t} ; \quad B=\left(\frac{\partial f}{\partial x^{*}}\right)^{2}+\left(\frac{\partial f}{s \partial z^{*}}\right)^{2} ; \\
a_{x^{*}}=a \cos \omega=-\frac{1}{B_{1}} \frac{\partial f}{\partial x^{*}} \frac{\partial f}{\partial t} ; \quad a_{z^{*}}=a \sin \omega=-\frac{1}{B_{1} s} \frac{\partial f}{\partial z^{*}} \frac{\partial f}{\partial t}
\end{gathered}
$$

where $\omega$ is the angle between the $0 x$ axis and the disturbance propagation direction in the $x z$ plane, the expression for $N$ in (6) takes the form:

$$
N=\frac{\gamma-1}{2} \int_{0}^{\infty} \frac{\left(g-u^{2}-w^{2}\right)^{2} d \eta}{(a-u \cos \omega-w \sin \omega)^{2}}-\int_{0}^{\infty}\left(g-u^{2}-w^{2}\right) d \eta .
$$


The characteristic surface $f(x, z, t)=0$ is determined by the condition $N=0$. This equation determines the mean velocity of disturbance propagation if the velocity and enthalpy profiles are known. Thus, if the surface $f(x, z, t)=0$ exists, then it divides the flow into regions in which disturbances can and cannot travel upstream. In particular case, this condition gives an expression from [5-7].

\section{RESULTS OF THE NUMERICAL ANALYSIS}

For determining the disturbance propagation velocity, it is necessary to know the velocity and enthalpy profiles in the boundary layer. These profiles can be found by solving stationary 3D boundary-layer Eqs. (2). The self-similar solution of equations for $s=1, \gamma=1.4$, and $\sigma=1$ was used for determining the vector $\vec{a}=\left(a_{x^{*}}, a_{z^{*}}\right)$ as a function of the temperature factor $g_{w}$. In this work, the radiation pattern of the disturbance propagation velocities is defined for $g_{w}=0.05,0.1,0.2,0.3,0.4$ and different $z$. Figure 2 presents the plots of the velocities of the upstream disturbance propagation for $z=0, \omega=180^{\circ}$ on the parameter ranges $g_{w} \in[0.05,0.4]$. An increase of $g_{w}$ leads to essential increase of the disturbance propagation velocity.

In Fig. 3, the velocities of the upstream disturbance propagation on the coordinate $z$ are plotted.

In Fig. 4, the radiation pattern of the disturbance propagation velocities is presented for $z=0$.

In Fig. 5, the radiation patterns of the disturbance propagation velocities are presented for $g_{w}=0.05$ and 0.4 .

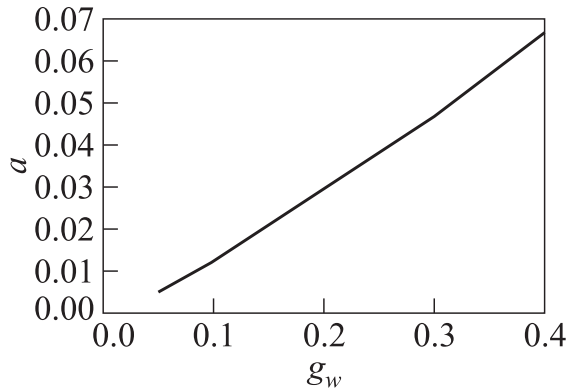

Figure 2 Velocity of the upstream disturbance propagation for $z=0$ and $\omega=180^{\circ}$

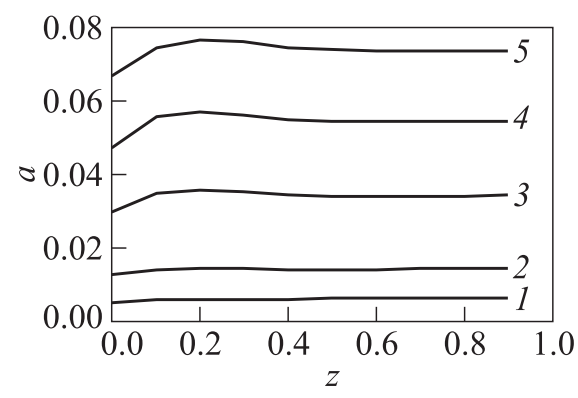

Figure 3 Velocity of the upstream disturbance propagation on the coordonate $z: 1-g_{w}=0.05 ; 2-0.1 ; 3-$ $0.2 ; 4-0.3 ;$ and $5-g_{w}=0.4$ 


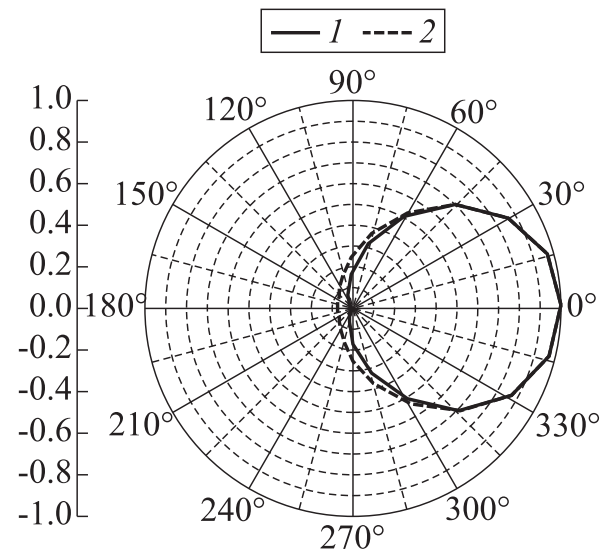

Figure 4 Radiation pattern of the disturbance propagation velocities; $z=0: 1-$ $g_{w}=0.05 ;$ and $2-g_{w}=0.4$

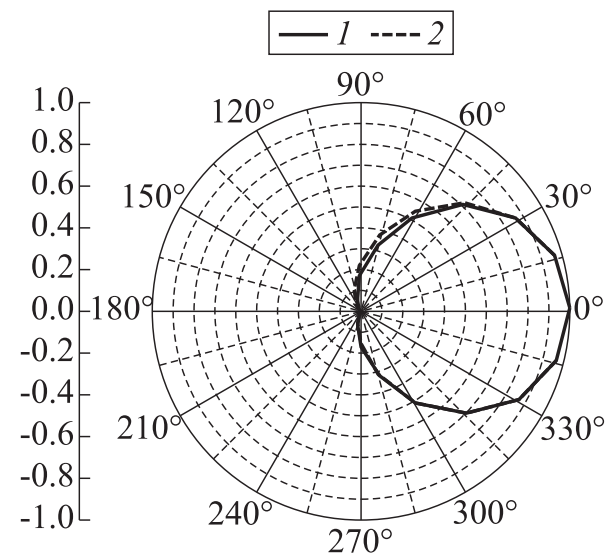

(a)

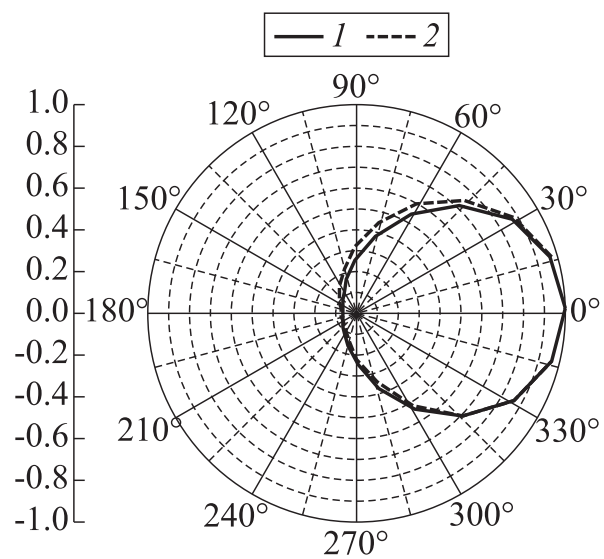

(b)

Figure 5 Radiation patterns of the disturbance propagation velocities for $g_{w}=$ $0.05(a)$ and $0.4(b): 1-z=0$; and $2-z=-0.9$

Qualitatively, the radiation patterns (see Figs. 4 and 5) are similar to the radiation pattern obtained for a semiinfinite plate for yaw angle [7].

\section{ACKNOWLEDGMENTS}

The work was performed with support of the Russian Foundation for Basic Research (No. 07-01-00349a) and ISTC. 


\section{REFERENCES}

1. Hayes, W. D., and R.F. Probstein. 1959. Hypersonic flow theory. New York: Academic Press.

2. Neiland, V. Ya., V. V. Bogolepov, G. N. Dudin, and I. I. Lipatov. 2008. Asymptotic theory of supersonic viscous gas flows. Amsterdam-London-New York: Elsevier.

3. Wang, K. C. 1971. On the determination of the zones of influence and dependence for three-dimensional boundary-layer equations. J. Fluid. Mech. 48(2):397.

4. Wang, K.C. 1975. Aspects of multitime initial-value problem originating from boundary layer equations. Phys. Fluids 18(8):951.

5. Pearson, H., J.B. Holliday, and S. F. Smith. 1958. Theory of the cylindrical ejector propelling nozzle. J. Roy. Aeron. Soc. 62(574):746.

6. Neiland, V. Ya. 1974. On the theory of interaction with boundary layer for separated two-dimensional and three-dimensional flows. Part 1. Three-dimensional flows. Uch. Zap. TsAGI 5(2):70.

7. Lipatov, I. I. 1995. Disturbance propagation in supersonic boundary layers. IUTAM Symposium Proceeding. Kluwer. 